\title{
iD-Sets and Associated Separation Axioms
}

\author{
Marwan A. Jardo \\ Department of Mathematics \College of Education \\ University of Mosul
}

Received

24/09/2013

\section{Accepted}

05/03/2014

\begin{abstract}
الخلاصة
في هذا البحث، قدمنا المجاميع من النمط-iDi و التي تعتمد على المجاميع المفتوحة من

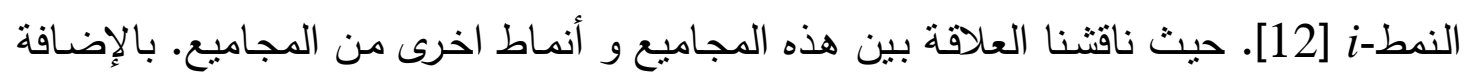

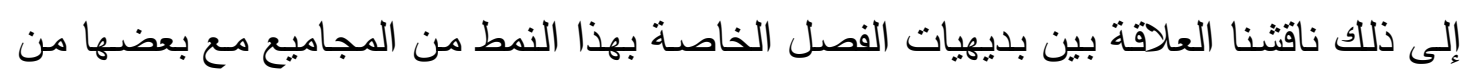

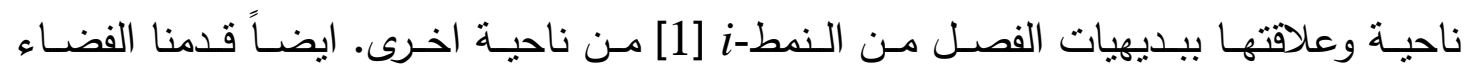

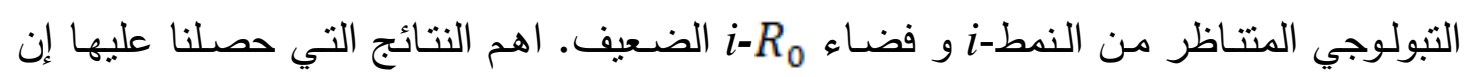
الفضاء المتتاظر من النمط-i-T الموسع تبولوجياً للمجاميع المفتوحة من النمط-i إذا وفقط اذا كان فضاء
\end{abstract}

\section{Abstract}

In this paper, we introduce $i D$-sets which are depends on i-open sets [12]. Where we discussed the relationship between this sets and other types of sets, In addition, we discussed the relationship between the separation axioms for this type of sets with one hand and their relationship with i-separation axioms [1] on the other hand. Also we introduce i-symmetric typological space and the weakly $i-R_{0}$ space. The most important results that we have obtained $i$-symmetric topological space which topologically extended for i-open sets will be $i-T_{1}$ space if and only if it is $i-T_{0}$ Space.

\section{Introduction}

In 1982, Tong [14] introduced the notion of $D$-sets and used these sets to introduce a separation axioms $D_{1}$ which is strictly between $T_{0}$ and $T_{1}$. In 1975, Maheshwari and Prasad [9] introduce new separation axioms semi- $T_{0}$, semi $T_{1}$ and semi- $T_{2}$ by using semi-open sets due to Levine [8]. Borsan [2] and Caldas [3] introduced the notions of $s D$-sets and separation axioms $s D_{1}$ which is strictly between semi- $T_{0}$ and semi- $T_{1}$. In 1980, Maheshwari and Thakur [10] introduced $\alpha-T_{2}$. In 1993, Maki, Devi 
and Balachandran [11] introduced $\alpha-T_{0}, \alpha-T_{1}$, by using $\alpha$-open sets due to Caldas, Georgiou and Jafari introduced the notion of $\alpha D$-sets and separation axioms $\alpha D_{1}$ which is strictly between $\alpha$ - To and $\alpha$ - T. Jardo in [6] introduced new separation axioms $i-T_{0}$ and $i-T_{1}$ and Askander [1] introduced $i$ - $T_{2}$ by using $i$-open sets. In this paper, we introduce $i D$-sets which are depends on i-open sets [12]. Where we discussed the relationship between this sets and other types of sets, In addition, we discussed the relationship between the separation axioms for this type of sets with one hand and their relationship with i-separation axioms [1] on the other hand. Also we introduce $i$-symmetric typological space and the weakly $i-R_{0}$ space. The most important results that we have obtained isymmetric topological space which topologically extended for $i$-open sets will be $i-T_{1}$ space if and only if it is $i-T_{0}$ Space.

\section{Preliminaries}

Throughout this paper, by $(X, \tau)$ and $(Y, \sigma)$ (or $X$ and $Y$ ) we always mean topological spaces. A subset $A$ of a topological space $(X, \tau)$ is called $\alpha$-open [13] (res. Semi-open [8] and $i$-open [12]). If $A \subseteq \operatorname{int}(\operatorname{cl}(\operatorname{int}(A)))$ (resp. $A \subseteq \operatorname{cl}(\operatorname{int}(A))$ and $A \subseteq \operatorname{cl}(A \cap G)$, where $\exists G \in \tau$ and $G \neq X, \phi)$. The complement of an $\alpha$-open (resp. Semi-open and $i$-open) set is called $\alpha$-closed [13] (resp. Semi-closed [4] and $i$-closed [12]). By $i O(X)$ (resp. $i C(X)$ ) we denote the family of all $i$-open (resp. $i$ closed) sets of $X$. The intersection of all $i$-closed subsets of $X$ containing $A$ is called the $i$-closure [12] of $A$, denoted by $\operatorname{cli}(A)$. A subset $A$ is $i$ closed if $A=\operatorname{cli}(A)$. Recall that from [12] if $(X, \tau)$ is a topological space then $X$ is called topologically extended for $i$-open sets (briefly, T. E. I.) if the family of all $i$-open sets of $X$ is a topology on $X$.

Lemma 2.1 [6] Let $(X, \tau)$ be a topological space and $A \subseteq X$, then $\operatorname{cli}(A)$ is $i$-closed, i.e. $\operatorname{cli}(\operatorname{cli}(A))=\operatorname{cli}(A)$.

Lemma 2.2 [6] For subsets, $A, B$ of a topological space $(X, \tau)$, the following statement hold:

1) $A \subseteq \operatorname{cli}(A)$,

2) If $A \subseteq B$, then $\operatorname{cli}(A) \subseteq \operatorname{cli}(B)$.

\section{Lemma 2.3}

1) Every open set in a topological space is an $\alpha$-open set [13]. 
2) Every $\alpha$-open set in a topological space is a semi-open set [5].

3) Every semi-open set in a topological space is an $i$-open set [6].

Definition 2.1 A subset $A$ of a topological space $(X, \tau)$ is called:

a) $D$-set [14] if there are $U, V \in \tau$ such that $U \neq X$ and $A=U \backslash V$,

b) $\alpha D$-set [14] if there are $U, V \in \alpha O(X)$ such that $U \neq X$ and $A=U \backslash V$,

c) $s D$-set [14] if there are $U, V \in S O(X)$ such that $U \neq X$ and $A=U \backslash V$.

Definition 2.2 A topological space $(X, \tau)$ is said to be:

a) $i-T_{0}$ [6] if for any distinct pair of points in $X$, there is an $i$-open set containing one of the points but not the other.

b) $i-T_{1}[6]$ if for any distinct pair of points $x$ and $y$ in $X$, there is an $i$ open set $U$ in $X$ containing $x$ but not $y$ and an $i$-open set $V$ in $X$ containing $y$ but not $x$.

c) $i-T_{2}[1]$ if for any distinct pair of points $x$ and $y$ in $X$, there is an $i$ open sets $U$ and $V$ in $X$ containing $x$ and $y$ respectively, such that $U \cap V=\phi$.

\section{3. $i \mathrm{D}-$ Sets and Associated Separation Axioms}

Definition 3.1 A subset $A$ of a topological space $(X, \tau)$ is called an $i$ deference (briefly, $i D$-set) if there are $U, V \in i O(X)$ such that $U \neq X$ and $A=U \backslash V$.

Remark 3.1 Every $i$-open set $U$ different from $X$ is an $i D$-set, if $A=U$ and $V=\phi$. But, the converse is false as the next example shows.

Example 3.1 Let $(X, \tau)$ be a topological space such that $X=\{a, b, c\}$ and $\tau=\{\phi, X,\{a, b\}\}$. Then $A=\{c\}$ is an $i D$-set but it is not $i$-open. In fact, since $i O(X)=\{\phi, X,\{a\},\{b\},\{a, b\},\{a, c\},\{b, c\}\}$, then $U=\{a, c\} \neq X$ and $V=\{a\}$ are $i$-open sets in $X$. Since $A=U \backslash V=\{a, c\} \backslash\{a\}=\{c\}$ is an $i D$-set but it is not $i$-open set.

\section{Theorem 3.1}

a) Every $D$-set is an $i D$-set,

b) Every $\alpha D$-set is an $i D$-set,

c) Every $s D$-set is an $i D$-set.

Proof: Clearly, since every open ( $\alpha$-open, semi-open) set is an $i$-open set.

The converse of Theorem 3.1 is not true. Indeed

Example 3.2 Let $X$ and $\tau$ be as in the Example 3.1, then $O(X)=\alpha O(X)=s O(X)=\{\phi, X,\{a, b\}\}$. Hence $A=\{c\}$ is an $i D$-set but it is not $D$-set, $\alpha D$-set and $s D$-set. 
Definition 3.2 A topological space $(X, \tau)$ is called $i D_{0}$ if for any pair of distinct points $x$ and $y$ of $X$ there exists an $i D$-set of $X$ containing $x$ but not $y$ or an $i D$-set of $X$ containing $y$ but not $x$.

Definition 3.3 A topological space $(X, \tau)$ is called $i D_{1}$ if for any pair of distinct points $x$ and $y$ of $X$ there exists an $i D$-set Aof $X$ containing $x$ but not $y$ and an $i D$-set $B$ of $X$ containing $y$ but not $x$.

Definition 3.4 A topological space $(X, \tau)$ is called $i D_{2}$ if for any pair of distinct points $x$ and $y$ of $X$ there exists disjoint $i D$-sets $A$ and $B$ of $X$ containing $x$ and $y$ respectively.

\section{Remark 3.2}

(i) If $(X, \tau)$ is $i-T_{k}$, then $(X, \tau)$ is $i-T_{k-1}, k=1,2$.

(ii) If $(X, \tau)$ is $i-T_{k}$, then $(X, \tau)$ is $i-D_{k}, k=0,1,2$.

(iii)If $(X, \tau)$ is $i-D_{k}$, then $(X, \tau)$ is $i-D_{k-1}, k=1,2$.

Theorem 3.2 For a topological space $(X, \tau)$ the following statement hold:

(1) $(X, \tau)$ is $i-D_{0}$ if and only if it is $i-T_{0}$,

(2) $(X, \tau)$ is $i-D_{1}$ if and only if it is $i-D_{2}$.

Proof. (1) The sufficiency is stated in Remark 3.2(ii). To prove necessity, let $(X, \tau)$ be $i-D_{0}$. Then for each distinct pair $x, y \in X$, at least one of $x, y$, say $x$, belongs to an $i D$-set $G$ where $y \notin G$. Let $G=U_{1} \backslash U_{2}$ such that $U_{1} \neq X$ and $U_{1}, U_{2} \in i O(X)$. Then $x \in U 1$. For $y \notin G$ we have two cases:

(a) $y \notin U_{1}$; (b) $y \in U_{1}$ and $y \in U_{2}$.

In case (a), $x \in U_{1}$ but $y \notin U_{1}$;

In case (b), $y \in U_{2}$ but $x \notin U_{2}$. Hence $X$ is $i-T_{0}$.

(2) Sufficiency. Remark 3.2(iii). necessity, let $(X, \tau)$ be $i-D_{1}$. Then for each distinct pair $x, y \in X$, we have $i D$-set $G_{1}, G_{2}$ such that $x \in G_{1}, y \notin G_{1} ; y \in G_{2}, x \notin G_{2}$. Let $G_{1}=U_{1} \backslash U_{2}, G_{2}=U_{3} \backslash U_{4}$. By $x \notin G_{2}$, it follows that either $x \notin U_{3}$ or $x \in U_{3}$ and $x \in U_{4}$. Now we consider two cases:

(1) $x \notin U_{3}$. By $y \notin G_{1}$ we have two sub cases:

(a) $y \notin U_{1}$. By $x \in U_{1} \backslash U_{2}$, it follows that $x \in U_{1} \backslash\left(U_{2} U U_{3}\right)$ and by $y \in U_{3} \backslash U_{4}$, we have $y \in U_{3} \backslash\left(U_{1} \cup U_{4}\right)$.

Hence, $U_{1} \backslash\left(U_{2} \cup U_{3}\right) \cap U_{3} \backslash\left(U_{1} \cup U_{4}\right)=\phi$.

(b) $y \in U_{1}$ and $y \in U_{2}$. We have $x \in U_{1} \backslash U_{2}, y \in U_{2}$, $\left(U_{1} \backslash U_{2}\right) \cap U_{2}=\phi$.

(2) $x \in U_{3}$ and $x \in U_{4}$. We have $x \in U_{3} \backslash U_{4}, y \in U_{4}$, $\left(U_{3} \backslash U_{4}\right) \cap U_{4}=\phi$.

Therefore $X i-D_{2}$. 
Corollary 3.1 if $(X, \tau)$ is $i-D_{1}$, then it is $i-T_{0}$.

Proof. By Remark 3.2 and Theorem 3.2.

The converse of corollary 3.1 is not true. Indeed

Example 3.2 Let $(X, \tau)$ be a topological space such that $X=\{a, b\}$ and $\tau=\{\phi, X,\{a\}\}$. Clearly the space $(X, \tau)$ is $i-T_{0}$ but it is not $i-D_{1}$.

Theorem 3.3[6] A T. E. I. $X$ is $i-T_{0}$ if and only if for each pair of distinct points $x, y$ of $X, \operatorname{cli}(\{x\})=\operatorname{cli}(\{y\})$.

Theorem 3.4[6] A T. E. I. $X$ is $i-T_{1}$ if and only if the singletons are $i$ closed sets.

Definition 3.5 A set $U$ in a topological space $(X, \tau)$ is an $i$-neighborhood of a point $x$ if $U$ contains an $i$-open set $V$ such that $x \in V$.

Definition 3.6 A point $x \in X$ which has $X$ as the unique $i$-neighborhood is called an $i$-neat point. set $U$ in a topological space $(X, \tau)$ is an $i$ neighborhood of a point $x$ if $U$ contains an $i$-open set $V$ such that $x \in V$.

Theorem 3.5 For an $i-T_{0}$ topological space $(X, \tau)$ the following are equivalent:

(1) $(X, \tau)$ is $i-D_{1}$,

(2) $(X, \tau)$ has no $i$-neat point.

Proof. $(1) \rightarrow(2)$. Since $(X, \tau)$ is $i-D_{1}$, then each point $x$ of $X$ is contained in an $i D$-set $O=U \backslash V$ and thus in $U$. By definition $U \neq X$. This implies that $x$ is not an $i$-neat point.

(2) $\rightarrow(1)$. If $X$ is $i-T_{0}$, then for each pair of distinct points $x$ and $y$ of $X$, at least one of them, $x$ (say) has an $i$-neighborhood $U$ containing $x$ and not $y$. Thus $U$ which is different from $X$ is an $i D$-set. If $X$ has no $i$-neat point, then $y$ is not an $i$-neat point. This means that there exists an $i$ neighborhood $V$ of $y$ such that $V \neq X$. Thus $y \in(V \backslash U)$ but not $x$ and $V \backslash U$ is an $i D$-set. Hence $X$ is $i D_{1}$.

Remark 3.3 it is clear that an $i-T_{0}$ topological space $(X, \tau)$ is not $i D_{1}$ if and only if there is a unique $i$-neat point in $X$. It is unique because if $x$ and $y$ are both $i$-neat point in $X$, then at least one of them say $x$ has an $i$ neighborhood $U$ containing $x$ but not $y$. But this is contradiction since $U \neq X$.

Definition 3.7 A topological space $(X, \tau)$ is $i$-symmetric if for every $x$ and $y$ in $X, x \in \operatorname{cli}(\{y\})$ implies $y \in \operatorname{cli}(\{x\})$. 
Definition 3.8[6] A subset $A$ of a topological space $(X, \tau)$ is called an $i$ generalized closed set (briefly, ig-closed) if $\operatorname{cli}(A) \subseteq U$ whenever $A \subseteq U$ and $U$ is an $i$-open set of $X$.

Lemma 3.1[6] Every $i$-closed set is ig-closed.

Theorem 3.6 A T. E. I. $X$ is $i$-symmetric if and only if $\{x\}$ is $i g$-closed set for each $x \in X$.

Proof. Assume that $x \in \operatorname{cli}(\{y\})$ but $y \notin \operatorname{cli}(\{x\})$. This means that $[\operatorname{cli}(\{x\})]^{c}$ contains $y$. Therefore the set $\{y\}$ is a subset of $[\operatorname{cli}(\{x\})]^{c}$. This implies that $\operatorname{cli}(\{y\})$ is a subset of $[\operatorname{cli}(\{x\})]^{c}$. Now $[\operatorname{cli}(\{x\})]^{c}$ contains $x$ which is a contradiction.

Conversely, suppose that $\{x\} \subseteq E \in i O(X)$ but $\operatorname{cli}(\{x\})$ is not a subset of $E$. This means that $\operatorname{cli}(\{x\})$ and $E^{c}$ are not disjoint. Let $y \in \operatorname{cli}(\{x\}) \cap E^{c}$. We have $x \in \operatorname{cli}(\{y\})$ which is a subset of $E^{c}$ and $x \notin E$. But this is a contradiction.

Corollary 3.2 If A T. E. I. $X$ is $i-T_{1}$ space, then it is $i$-symmetric.

Proof. Let $X$ be an $i-T_{1}$ space, then singleton sets are $i$-closed set (Theorem 3.4) and therefore ig-closed set (Lemma 3.1). By (Theorem 3.6) $X$ is $i$-symmetric.

Corollary 3.3 For a T. E. I. $X$ the following are equivalent:

(1) $X$ is $i-T_{0}$ and $i$-symmetric,

(2) $X$ is $i-T_{1}$.

Proof. By Corollary 3.2 and Remark 3.2 (i) it suffices to prove only $(1) \rightarrow(2)$. Let $x \neq y$ and by $i-T_{0}$, we may assume that $x \in G_{1} \subseteq\{y\}^{c}$ for some $G_{1} \in i O(X)$. Then $x \notin \operatorname{cli}(\{y\})$ and hence $y \notin \operatorname{cli}(\{x\})$. There exists $G_{2} \in i O(X)$ such that $y \in G_{2} \subseteq\{x\}^{c}$. Hence $X$ is $i-T_{1}$ space.

Theorem 3.7 Let $X$ is $i$-symmetric T. E. I. the following are equivalent:

(1) $X$ is $i-T_{0}$,

(2) $X$ is $i-D_{1}$,

(3) $X$ is $i-T_{1}$.

The proof is straightforward and hence omitted.

We recall the following:

Definition 3.9[12] A function $f:(X, \tau) \rightarrow(Y, \sigma)$ is $i$-irresolute if the inverse image of each $i$-open set is $i$-open.

Theorem 3.8 if $f:(X, \tau) \rightarrow(Y, \sigma)$ is an $i$-irresolute surjective function and $E$ is an $i D$-set in $Y$, then the inverse image of $E$ is an $i D$-set in $X$. 
Proof. Let $E$ be an $i D$-set in $Y$, then there are $i$-open sets $U_{1}$ and $U_{2}$ in $Y$ such that $E=U_{1} \backslash U_{2}$ and $U_{1} \neq Y$. By the irresoluteness of $f, f^{-1}\left(U_{1}\right)$ and $f^{-1}\left(U_{2}\right)$ are $i$-open sets in $X$.since $U_{1} \neq Y$, we have $f^{-1}\left(U_{1}\right) \neq X$. Hence $f^{-1}(E)=f^{-1}\left(U_{1}\right) \backslash f^{-1}\left(U_{2}\right)$ is an $i D$-set in $X$.

Theorem 3.9 if $(Y, \sigma)$ is $i D_{1}$ and $f:(X, \tau) \rightarrow(Y, \sigma)$ is $i$-irresolute and bijective, then $(X, \tau)$ is $i D_{1}$ space.

Proof. Suppose that $Y$ is $i D_{1}$ space. Let $x$ and $y$ be any pair of distinct points in $X$. Since $f$ is injective and $Y$ is $i D_{1}$, then there exist $i D$-sets $G_{f(x)}$ and $G_{f(y)}$ of $Y$ containing $f(x)$ and $f(y)$, respectively, such that $f(y) \notin G_{f(x)}$ and $f(x) \notin G_{f(y)}$. By Theorem 3.8, $f^{-1}\left(G_{f(x)}\right)$ and $f^{-1}\left(G_{f(y)}\right)$ are $i D$-sets in $X$ containing $x$ and $y$, respectively, This implies that $X$ is an $i D_{1}$ space.

Definition 3.10 A function $f:(X, \tau) \rightarrow(Y, \sigma)$ is called always $i$-open if the image of each $i$-open set in $X$ is $i$-open in $Y$.

Theorem 3.10 Let $X$ be an arbitrary space, $R$ an equivalence relation in $X$ and $h: X \rightarrow X \backslash R$ the identification map. If $R \subseteq X \times X$ is $i$-closed in $X \times X$ and $h$ is an always $i$-open map. Then $X \backslash R$ is $i-T_{2}$.

Proof. Let $h(x), h(y)$ be distinct members of $X \backslash R$. Since $x$ and $y$ are not related, $R \subseteq X \times X$ is $i$-closed in $X \times X$. There are $i$-open sets $U$ and $V$ such that $x \in U, y \in V$ and $U \times V \subseteq R^{c}$. Thus $h(U), h(V)$ are disjoint and also $i$-open in $X \backslash R$ since $h$ is always $i$-open.

Theorem 3.11 A topological space $(X, \tau)$ is $i D_{1}$ if for each pair of distinct points $x, y \in X$, there exists an $i$-irresolute surjective function $f:(X, \tau) \rightarrow(Y, \sigma)$, where $(Y, \sigma)$ is an $i D_{1}$ space such that $f(x)$ and $f(y)$ are distinct.

Proof. Necessity. For every pair of distinct points of $X$, it suffices to take the identity function of $X$. Sufficiency, Let $x$ and $y$ be any pair of distinct points in $X$. By hypothesis, there exists $i$-irresolute surjective function $f$ of a space $(X, \tau)$ onto a $i D_{1}$ space $(Y, \sigma)$ such that $\mathrm{f}(x) \neq f(y)$. Therefore, there exists $i D$-sets $G_{f(x)}$ and $G_{f(y)}$ in $Y$ such that $f(x) \in G_{f(x)}$ and $f(y) \in G_{f(y)}$. Since $f$ is $i$-irresolute and surjective, by theorem 3.8, $f^{-1}\left(G_{f(x)}\right)$ and $f^{-1}\left(G_{f(y)}\right)$ are $i D$-sets in $X$ containing $x$ and $y$, respectively. Therefore the space $X$ is an $i D_{1}$ space.

Lemma 3.2 Let $(X, \tau)$ be a topological space. If $A, B \in i O(X)$, then $A \times B \in i O(X)$. 
Proof. Let $A, B \in i O(X)$, then $A \subseteq \operatorname{cl}\left(A \cap G_{1}\right)$ and $B \subseteq \operatorname{cl}\left(B \cap G_{2}\right)$ where $G_{1}, G_{2} \in \tau$ and $G_{1}, G_{2} \neq \phi, X$.

Now, $A \times B \subseteq \operatorname{cl}\left(A \cap G_{1}\right) \times \operatorname{cl}\left(B \cap G_{2}\right)=\operatorname{cl}\left(\left(A \cap G_{1}\right) \times\left(B \cap G_{2}\right)\right)=$ $\operatorname{cl}\left((A \times B) \cap\left(A \times G_{2}\right) \cap\left(B \times G_{1}\right) \cap\left(G_{1} \times G_{2}\right)\right)=$ $\operatorname{cl}\left(\left[(A \times B) \cap\left(G_{1} \times G_{2}\right)\right] \cap\left[\left(A \times G_{2}\right) \cap\left(B \times G_{1}\right)\right]\right) \subseteq \operatorname{cl}\left((A \times B) \cap\left(G_{1} \times G_{2}\right)\right.$ where $G_{1} \times G_{2}$ is open set [8], and $G_{1} \times G_{2} \neq \phi, X$.

Hence, $A \times B \in i O(X)$.

Theorem 3.12 Let $X$ be a T. E. I., then the following properties are equivalent:

(1) $X$ is $i-T_{2}$,

(2) Let $x \in X$. For each $y \neq x$, there exists an $i$-open set $U$ such that $x \in U$ and $y \notin \operatorname{cli}(U)$,

(3) For each $x \in X, \cap\{\operatorname{cli}(U) ; U \in i O(X)$ and $x \in U\}=\{x\}$,

(4) The diagonal $\Delta=\{(x, x) ; x \in X\}$ is $i$-closed in $X \times X$.

Proof. (1) $\rightarrow(2)$. Let $x \in X$ and $y \neq x$. Then there are disjoint $i$-open sets $U$ and $V$ such that $x \in U$ and $y \in V$. Clearly, $V^{c}$ is $i$-closed set, $\operatorname{cli}(U) \subseteq V^{c}, y \notin V^{c}$ and therefore $y \notin, \operatorname{cli}(U)$.

(2) $\rightarrow(3)$. If $y \neq x$, then there exists an $i$-open set $U$ such that $x \in U$ and $y \notin \operatorname{cli}(U)$. So $y \notin \cap\{\operatorname{cli}(U) ; U \in i O(X)$ and $x \in U\}$.

(3) $\rightarrow(4)$. We will prove that $\Delta^{c}$ is $i$-open. Let $(x, y) \notin \Delta$. Then $y \neq x$ and since $\cap\{\operatorname{cli}(U) ; U \in i O(X)$ and $x \in U\}=\{x\}$, there is some $U \in i O(X)$ where $x \in U$ and $y \notin \operatorname{cli}(U)$. Since $U \cap(\operatorname{cli}(U))^{c}=\phi, U \times(\operatorname{cli}(U))^{c}$ is an $i$-open set such that $(x, y) \in U \times(\operatorname{cli}(U))^{c} \subseteq \Delta^{c}$.

(4) $\rightarrow(1)$. If $y \neq x$, then $(x, y) \notin \Delta$ and thus there exists $i$-open sets $U$ and $V$ such that $(x, y) \in U \times V$ and $U \times V \cap \Delta=\phi$. Clearly, for the $i$-open sets $U$ and $V$ we have, $x \in U$ and $y \in V$ and $U \cap V=\phi$.

\section{Weakly $i-R_{0}$ spaces}

Definition 4.1 Let $A$ be a subset of a topological space $(X, \tau)$. The $i$ kernel of $A$, denoted by $i-\operatorname{ker}(A) \quad$ is defined to be the set $i-\operatorname{ker}(A)$ $=\cap\{O \in i O(X) ; A \subseteq O)\}$.

Lemma 4.1 Let $X$ be a T. E. I. and $A \subseteq X, x \in X$. Then $i-\operatorname{ker}(A)=$ $\{x \in X ; \operatorname{cli}(\{x\}) \cap A \neq \phi\}$.

Proof. Let $x \in i-\operatorname{ker}(A)$ and suppose $\operatorname{cli}(\{x\}) \cap A=\phi$. Hence $x \notin[\operatorname{cli}(\{x\})]^{c}$ which is an $i$-open set containing $A$. This is impossible, since $x \in i-\operatorname{ker}(A)$. Consequently, $\quad \operatorname{cli}(\{x\}) \cap A \neq \phi . \quad$ Next, let 
$\operatorname{cli}(\{x\}) \cap A \neq \phi$ and suppose that $x \notin i-\operatorname{ker}(A)$. Then, there exists an $i$ open $G$ containing $A$ and $\notin G$. Let $y \in \operatorname{cli}(\{x\}) \cap A$. Hence, $G$ is an $i$ neighborhood of $y$ which $\notin G$. By this contradiction $x \in i-\operatorname{ker}(A)$.

5. Definition 4.2 A topological space $(X, \tau)$ is said to be Weakly $i-R_{0}$ if $\bigcap_{x \in X} \operatorname{cli}(\{x\})=\phi$.

Theorem 4.1 A topological space $(X, \tau)$ is weakly $i-R_{0}$ if and only if $i-\operatorname{ker}(\{x\}) \neq X$ for every $x \in X$.

Proof. Suppose that the space $(X, \tau)$ is weakly $i-R_{0}$. Assume that there is a point $y$ in $X$ such that $i-\operatorname{ker}(\{y\})=X$. Then $y \notin O$ which $O$ is proper $i-$ open set of $X$. This implies that $y \in \bigcap_{x \in X} \operatorname{cli}(\{x\})$. But this is a contradiction. Now assume that $i-\operatorname{ker}(\{x\}) \neq X$ for every $x \in X$. If there exists a point $y$ in $X$ such that $y \in \bigcap_{x \in X} \operatorname{cli}(\{x\})$, then every $i$-open set containing $y$ must contain every point of $X$. This implies that the space $X$ is the unique $i$-open set containing $y$. Hence, $i-\operatorname{ker}(\{y\})=X$ which is a contradiction. Therefore $(X, \tau)$ is weakly $i-R_{0}$.

Definition 4.3 A function $f:(X, \tau) \rightarrow(Y, \sigma)$ is called always $i$-closed if the image of each $i$-closed set of $X$ is $i$-closed in $Y$.

Theorem 4.2 if $f:(X, \tau) \rightarrow(Y, \sigma)$ is an bijective always $i$-closed function and $X$ is weakly $i-R_{0}$, then $Y$ is weakly $i-R_{0}$.

Proof. Straightforward.

Theorem 4.3 If the topological space $X$ is weakly $i-R_{0}$ and $Y$ is any topological space, then the product $X \times Y$ is weakly $i-R_{0}$.

Proof. By showing that $\bigcap_{(x, y) \in X \times Y} \operatorname{cli}(\{(x, y)\})=\phi$ we are done. We have: $\bigcap_{(x, y) \in X \times Y} \operatorname{cli}(\{(x, y)\}) \subseteq \bigcap_{(x, y) \in X \times Y}(\operatorname{cli}(\{x\} \times \operatorname{cli}(\{y\}))=$ $\bigcap_{x \in X} \operatorname{cli}(\{x\}) \times \bigcap_{y \in Y} \operatorname{cli}(\{y\}) \subseteq \phi \times Y=\phi$.

\section{References}

[1] Askander S. W., "On $i$-Sepration Axioms", Mosul Univ., J. Education and Science, Education College, (accepted in 2013).

[2] Borsan D., "On semi-separation axioms", Babes-Bolyai Univ., Fac. Of Math., 4:107-114 (1986).

[3] Caldas M., A separation axioms between semi- $T_{0}$ and semi- $T_{1}$, Mem. Fac. Sci. Kochi Univ. Ser. Math. 181: 37-42 (1997).

[4] Crossley S. G. and Hildebrand S. K., semi-topological properties, Fund. Math., 74: 233-254 (1972).

[5]Dlaska K., Ergun N. and Ganster M., On the topology generated by semi-regular sets, Indian J. pure apple. Math., 25: 1163-1170 (1994). 
[6] Jardo M. A., On Generalized $i$-Closed Sets in Topological Spaces and Their Applications, M. Sc. Thesis, College of Education, Univ. of Mosul (2012).

[7] Keskin A. and Noiri T., On bD-sets and associated separation axioms, Bulletin of the Iranian Math. Sci., 1: 179-198 (2009).

[8] Levine N., Semi-open sets and semi-continuity in topological spaces, Amer. Math. Monthly, 70: 36-41 (1963).

[9] Maheshwari S. N. and Prasad R., Some new separation axioms, Ann. Soc. Sci. Bruxelles, 89: 395-407 (1975).

[10] Maheshwari S. N. and Thakur S. S., On $\alpha$-irresolute mapping, Tamkang J. Math., 11: 209-214 (1980).

[11] Maki H. Devi R. and Balachandran K., Generalized $\alpha$-closed sets in topology, Bull. Fukuoka Univ. Ed., 42: 13-21 (1993).

[12] Mohammed A. A. and Askander S. W., On $i$-open sets, U. A. E., Math. day conference, Amer. Univ. of Sharjah, April, 14, 2012.

[13] Njastad O., On some classes of nearly open sets, pacific J. Math., 15: $961-970$ (1965).

[14] Tong J., A separation axioms between $T_{0}$ and $T_{1}$, Ann. Soc. Sci. Bruxelles, 96: 85-90 (1982). 